\title{
Targeted capture enrichment followed by NGS: development and validation of a single comprehensive NIPT for chromosomal aneuploidies, microdeletion syndromes and monogenic diseases
}

George Koumbaris ${ }^{1}$, Achilleas Achilleos ${ }^{1}$, Michalis Nicolaou', Charalambos Loizides ${ }^{1}$, Kyriakos Tsangaras ${ }^{1}$, Elena Kypri ${ }^{1}$, Petros Mina ${ }^{1}$, Carolina Sismani ${ }^{2,3}$, Voula Velissariou ${ }^{1,4}$, Georgia Christopoulou, Pantelis Constantoulakis ${ }^{5}$, Emmanouil Manolakos ${ }^{6}$, Ioannis Papoulidis ${ }^{6}$, Danai Stambouli ${ }^{7}$, Marios loannides ${ }^{1}$ and Philippos Patsalis ${ }^{1 *}$

\begin{abstract}
Background: Non-invasive prenatal testing (NIPT) has been widely adopted for the detection of fetal aneuploidies and microdeletion syndromes, nevertheless, limited clinical utilization has been reported for the non-invasive prenatal screening of monogenic diseases. In this study, we present the development and validation of a single comprehensive NIPT for prenatal screening of chromosomal aneuploidies, microdeletions and 50 autosomal recessive disorders associated with severe or moderate clinical phenotype.

Results: We employed a targeted capture enrichment technology powered by custom TArget Capture Sequences (TACS) and multi-engine bioinformatics analysis pipeline to develop and validate a novel NIPT test. This test was validated using 2033 cell-fee DNA (cfDNA) samples from maternal plasma of pregnant women referred for NIPT and paternal genomic DNA. Additionally, 200 amniotic fluid and CVS samples were used for validation purposes. All NIPT samples were correctly classified exhibiting 100\% sensitivity (Cl 89.7-100\%) and 100\% specificity (Cl 99.8-100\%) for chromosomal aneuploidies and microdeletions. Furthermore, 613 targeted causative mutations, of which 87 were unique, corresponding to 21 monogenic diseases, were identified. For the validation of the assay for prenatal diagnosis purposes, all aneuploidies, microdeletions and point mutations were correctly detected in all 200 amniotic fluid and CVS samples.
\end{abstract}

Conclusions: We present a NIPT for aneuploidies, microdeletions, and monogenic disorders. To our knowledge this is the first time that such a comprehensive NIPT is available for clinical implementation.

Keywords: NIPT, Monogenic diseases, Cell-free DNA, Aneuploidies, Microdeletions

\footnotetext{
* Correspondence: p.patsalis@nipd.com

'NIPD Genetics Public Company Ltd, Neas Engomis 31, Nicosia 2409, Cyprus

Full list of author information is available at the end of the article
}

(c) The Author(s). 2019 Open Access This article is distributed under the terms of the Creative Commons Attribution 4.0 International License (http://creativecommons.org/licenses/by/4.0/) which permits unrestricted use, distribution, and reproduction in any medium, provided you give appropriate credit to the original author(s) and the source, provide a link to the Creative Commons license, and indicate if changes were made. The Creative Commons Public Domain Dedication waiver (http://creativecommons.org/publicdomain/zero/1.0/) applies to the data made available in this article, unless otherwise stated. 


\section{Background}

Until recently, prenatal screening for fetal aneuploidies relied on the measurement of maternal serum biochemical markers combined with fetal ultrasound markers. The discovery of cell-free fetal DNA (cffDNA) in maternal circulation prompted the development of noninvasive prenatal testing (NIPT), opening a new era in prenatal screening [1]. Since its introduction, different methods have been applied for the detection of fetal aneuploidies, mainly employing whole genome or targeted approaches combined with Next Generation Sequencing (NGS). The success of such methods has been highlighted in several clinical validation studies that demonstrate the ability for high aneuploidy detection rates [2-9]. This has led to the endorsement of NIPT by several professional bodies as a primary screening method regardless of the pregnancy risk status [10-12]. Furthermore, in the absence of specific markers, NIPT can be used for sex chromosomal aneuploidies and for select microdeletion syndromes [10].

For the non-invasive prenatal screening of monogenic diseases limited clinical utilization has been reported. Early studies were focused on disease detection or exclusion based on the presence of de novo or paternally inherited mutations respectively, using PCR approaches, allowing only for the screening of a limited number of monogenic diseases [13-15]. Recently, the advent of high precision and high throughput technologies such as NGS and digital PCR, catalyzed the development of higher sensitivity assays for the detection of monogenic diseases in cfDNA [16-21], most of them requiring the parental haplotype to interpret the fetal inheritance pattern [18, 22, 23]. Nevertheless, limited scalability, high cost and increased complexity in assay performance and data analysis rendered their clinical implementation challenging [24].

Recently, Luo et al. described in a proof of concept study the feasibility of integrating three tests in a single NIPT for the detection of aneuploidies, large copy number variants $(>20 \mathrm{Mb})$ and a limited number of single gene diseases using target capture enrichment. The authors employed a direct NIPT approach for screening of monogenic diseases, which is highly dependent on fetal fraction. Despite the promising results, the sensitivity of the assay was low, especially for the detection of point mutations in the cffDNA even in samples with high fetal fraction. As the authors conclude, additional technological optimizations are needed to increase the test's accuracy and a larger sample cohort is required for validation and determination of the analytical performance of the test prior to clinical implementation [25]. For the determination of the fetal risk for monogenic diseases, unlike Luo et al., we followed a conventional, fetal fraction independent prenatal screening approach by assessing the fetal risk based on the parental carrier status, in the same workflow as cffDNA analysis for aneuploidies and microdeletions.

Addressing the need of NIPT as prenatal screening for both chromosomal and monogenic diseases, we have developed and validated a new, single, comprehensive NIPT providing the fetal risk for these types of genetic conditions. By employing our existing hybrid capturebased technology with minor modifications [7] and multi-engine bioinformatics analysis pipeline, we present the development and validation of a comprehensive NIPT which offers prenatal screening for aneuploidies of chromosomes 13, 18, 21, X, Y, four microdeletion syndromes and 50 autosomal recessive monogenic disorders with severe or moderate clinical phenotype. The fetal risk for aneuploidies and microdeletions is provided based on the analysis of cffDNA present in maternal plasma, whilst the fetal risk for the autosomal recessive monogenic diseases is determined based on Mendelian law of inheritance by combining maternal and paternal carrier status information using cell free maternal DNA (cfmDNA) and paternal DNA respectively.

\section{Results}

The workflow of this novel comprehensive NIPT prenatal screening test for aneuploidies of 13, 18, 21, X, Y, four microdeletion syndromes and 50 single gene diseases, followed by prenatal diagnosis for these disorders in case of high risk pregnancy is described in Additional file 1. The cfDNA, consisting of cffDNA and cfmDNA, from pregnant women of at least 10 weeks of gestation and paternal genomic DNA $(n=2033)$, were simultaneously analyzed for the detection of chromosomal aneuploidies (trisomy 21, trisomy 18 and trisomy 13), sex chromosome aneuploidies (SCA) (monosomy X, Klinefelter syndrome, trisomy $\mathrm{X}, \mathrm{XYY}$, and XXYY), four microdeletion syndromes (1p36 deletion syndrome, Wolf-Hirschhorn syndrome, Smith-Magenis syndrome and 22q11.2 deletion syndrome) and point mutations for 50 autosomal recessive conditions (Additional file 2).

In the 2033 samples analyzed with this single comprehensive NIPT, we identified all samples correctly, exhibiting $100 \%$ sensitivity (CI 89.7-100\%) and 100\% specificity (CI 99.8-100\%) for chromosomal aneuploidies and microdeletion syndromes. Specifically, 22/ 22 trisomy 21, 4/4 trisomy $18,1 / 1$ trisomy 13 cases, $2 / 2$ SCAs and $5 / 5$ microdeletion syndromes (three cases of 22q11.2 deletion, one case of Wolf-Hirschhorn syndrome and one case of Smith-Magenis syndrome) were correctly classified (Table 1 ).

The results were in agreement with the invasive prenatal diagnosis results performed by the collaborating laboratories for all tested samples. 
Table 1 Assay validation on cfDNA and paternal samples

\begin{tabular}{|c|c|c|c|}
\hline Description & $\begin{array}{l}\text { cfDNA and biological } \\
\text { father DNA }\end{array}$ & $\begin{array}{l}\text { Confirmed invasive } \\
\text { prenatal diagnosis }\end{array}$ & Confirmed by Sange \\
\hline Total & 2033 & & \\
\hline Trisomy 21 & 22 & $22 / 22$ & - \\
\hline Trisomy 18 & 4 & $4 / 4$ & - \\
\hline Trisomy 13 & 1 & $1 / 1$ & - \\
\hline SCA & 2 & $2 / 2$ & - \\
\hline Microdeletion syndromes & 5 & $5 / 5$ & - \\
\hline Number of samples with no mutations & 1497 & & $496 / 496^{a}$ \\
\hline Number of samples with mutations & 536 & & - \\
\hline Number of mutations & 613 & & - \\
\hline Number of unique mutations & 87 & & $87 / 87$ \\
\hline
\end{tabular}

${ }^{a}$ All 496 targeted mutations were confirmed in 5 randomly selected normal (wild-type) samples

In the same assay, we identified 1497/2033 samples carrying none of the targeted mutations (wild type) and 536/ 2033 samples carriers of one or more of the targeted mutations (Table 1). In total, 613 targeted causative mutations were detected, of which 87 were unique (detected at least once), corresponding to 21 monogenic diseases (Table 1). All unique point mutations identified in this cohort were verified by Sanger sequencing using parental genomic DNA (Fig. 1b). All wild-type variants $(n=496)$ were confirmed in five randomly selected normal (wildtype) parental samples (Table 1).

To provide a comprehensive prenatal screening and diagnosis solution, non-invasive results are supplemented with a fetal tissue reflex test using amniotic fluid or CVS for all cases found as high risk for any of the tested conditions (Additional file 1). Therefore, a second retrospective validation study for prenatal diagnosis was performed on 200 amniotic fluid and CVS samples,

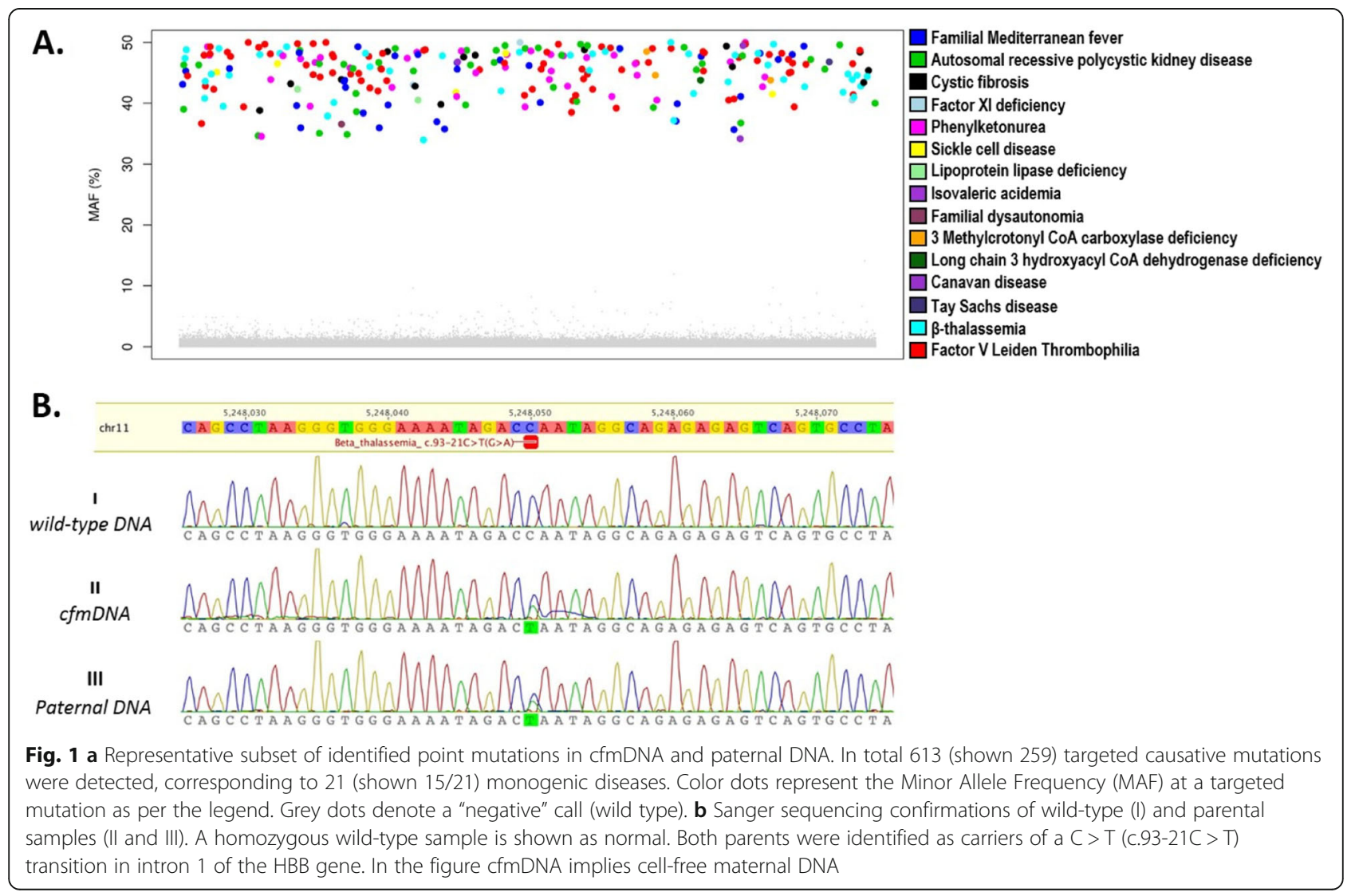


including 70 aneuploid and six microdeletion samples. Specifically, this targeted test correctly classified all 124 normal, 15 of which were found to carry causative mutations (heterozygous), corresponding to the 50 single gene diseases targeted in our panel (Table 2) and 76 abnormal samples with 100\% specificity (CI 97-100\%) and $100 \%$ sensitivity (CI 95-100\%) for aneuploidies and microdeletions (Table 2). The abnormal samples included 41 trisomy 21 , nine trisomy 18 , six trisomy 13,14 SCAs, four cases of 22q11.2 deletion and two WolfHirschhorn syndrome cases (Fig. 2).

\section{Discussion}

We have developed and validated a new comprehensive NIPT for fetal aneuploidies of chromosomes 13, 18, 21, $\mathrm{X}$ and $\mathrm{Y}$, four microdeletion syndromes (1p36, WolfHirschhorn, Smith-Magenis, 22q11.2) and a panel of 50 autosomal recessive single gene diseases, achieving very high sensitivity and specificity. Furthermore, we validated the assay for prenatal diagnosis using amniotic fluid and CVS, correctly classifying all normal and abnormal samples. As such, the newly developed single comprehensive test provides an extended and validated prenatal screening solution, including prenatal diagnosis for those pregnancies found as high risk for aneuploidies, microdeletions and monogenic diseases.

The benefits of non-invasive prenatal screening for aneuploidies and microdeletions have been thoroughly described, and NIPT is well accepted as an effective, efficient and cost effective prenatal screening for these syndromes [26]. The addition of monogenic diseases to NIPT within a single comprehensive prenatal screening test, as the one presented herein, provides added value to the field of prenatal screening and major benefits for the clinical practice and health care systems.

This single comprehensive NIPT is based on a validated targeted capture enrichment technology [7], offering technological advantages, high accuracy and short turnaround time (TAT). The test can be offered to all pregnant women from the 10th week of gestation onwards, including twin and IVF non-donor pregnancies, allowing timely and informed decisions regarding diagnosis, prevention and better clinical management of the pregnancy. This is the first time that a comprehensive NIPT is made available, setting the first step towards the clinical implementation of NIPT of chromosomal syndromes and monogenic diseases in the field of prenatal screening.

An inherent limitation of NIPT, irrespective of the approach taken, is the discordance between NIPT and prenatal diagnosis mainly due to confined placental mosaicism (CPM). As such, cases with CPM are potential sources of false-positive (FP) and false-negative (FN) results. However, considering the likelihood of the spontaneous abortion of trisomies 13 and 18 by the 12th week of pregnancy and term, and the incidence of trisomy 21 in the general population, the sensitivity and the specificity of NIPT is not considerably affected [7].

In its current form, the test screens for known pathogenic or likely pathogenic mutations covering in most cases higher than $70 \%$ detection rate and includes most common mutations reported for each condition (Additional file 4). As such, rare or familial mutations that may be associated with clinical phenotype are not covered by our existing panel. Recent studies have also stressed the importance of prenatal screening of dominant disorders caused by de novo or paternally inherited mutations, as these contribute to nearly $60 \%$ of severe postnatal monogenic disease [27]. Acknowledging the clinical relevance and importance of these conditions, work is underway to enrich the existing panel with rare mutations and also include autosomal dominant and Xlinked diseases, covering, in this way, all types of monogenic diseases in a single test.

Furthermore, the fetal risk for monogenic diseases is assessed based on the carrier status of the biological parents, therefore, pregnancies achieved using egg donation or surrogate pregnancies are excluded from the fetal risk assessment for monogenic diseases.

Based on the public databases the theoretical risk of being a carrier for at least one of the 50 single gene diseases included in this comprehensive NIPT is 1 in 3 , while in our cohort of 2033 samples it was found to be 1 in 4 . The actual risk for the fetus being affected by one of the 50 single gene diseases screened by this comprehensive NIPT in our cohort is 1 in 196. The actual total risk of the fetus being affected by one of the autosomal and sex chromosome aneuploidies, four microdeletion syndromes and 50 single gene diseases is 1 in 65 (Additional file 3). Based on the above estimates, all pregnancies matching the characteristics of our cohort

Table 2 Assay validation on amniotic fluid and CVS samples

\begin{tabular}{lll}
\hline Description & Normal samples & Abnormal samples \\
\hline Normal & $109 / 109$ & - \\
Aneuploidies of 13, 18, 21, X and Y & - & $70 / 70$ \\
Microdeletion syndromes & - & $6 / 6$ \\
Single gene diseases & - & $15 / 15^{\mathrm{a}}$ \\
\hline
\end{tabular}

${ }^{\mathrm{a}}$ Samples were found to be heterozygous (carriers) for targeted mutations 

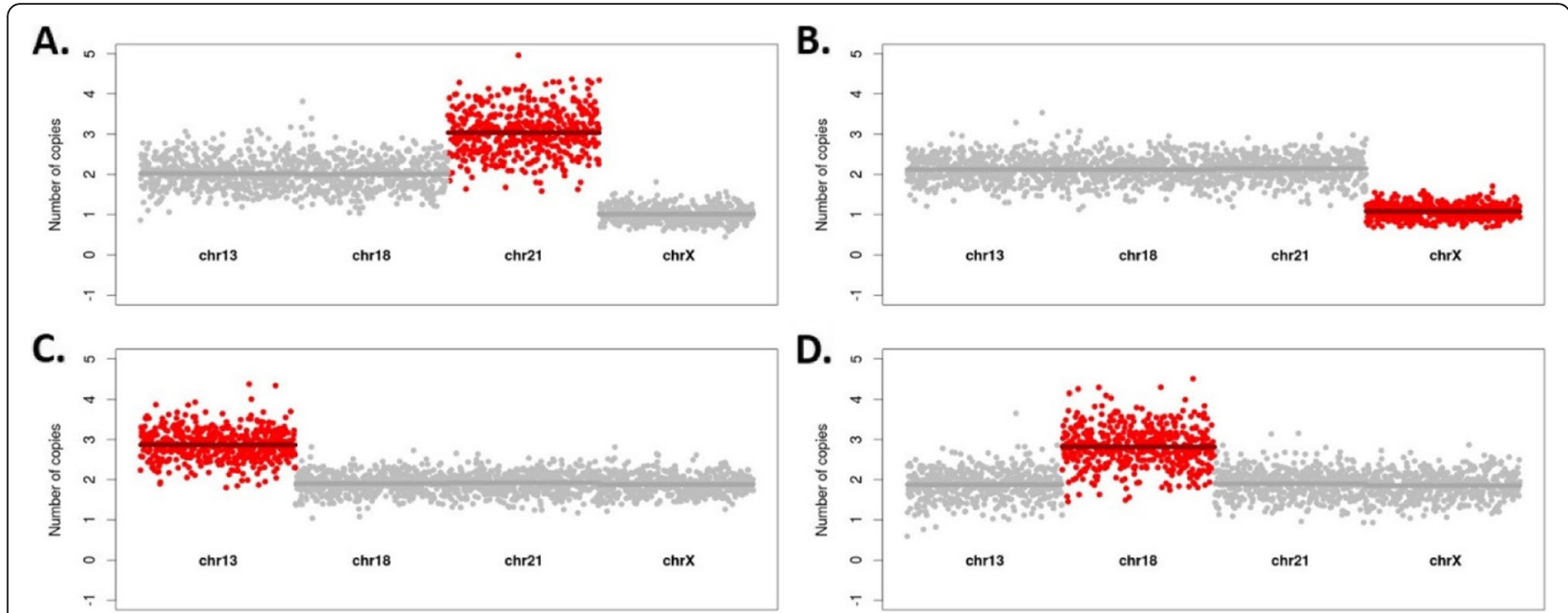

Fig. 2 Foetal abnormality detection in prenatal samples. In total, 200 prenatal samples (amniotic fluid and CVS) were subjected to prenatal diagnosis using the same single comprehensive assay. All normal and abnormal samples were correctly classified. The $x$-axis denotes the targeted chromosomes (chr 13, 18, 21, X). The y-axis represents the normalized read depth per TACS (dots). Red dots denote positive calls. The panels show the detection of a Trisomy 21, b Monosomy X, c Trisomy 13, d Trisomy 18

should be considered as high risk and screened for these conditions. In the absence of age-associated risk and available biochemical and ultrasound markers for the great majority of monogenic diseases, and given that any pregnancy having such a risk is considered as intermediate to high risk $[28,29]$, a first tier NIPT such as the one presented herein should be integrated as part of standard health care. Accordingly, being aware of the fetal risk, couples can take informed decisions regarding the clinical management of their pregnancy.

All monogenic diseases in our panel are associated with moderate to severe phenotype or require prenatal or neonatal intervention to improve and manage the pregnancy's outcome [30]. In accordance to professional bodies' opinions, we cover only pathogenic or likely pathogenic mutations, with overall disease detection frequency higher than $70 \%$ in most cases. Late onset diseases and mutations of unknown significance or mutations not sufficiently documented in the literature were excluded from our panel [31].

A recent population-based study describing couples' experience with carrier screening revealed that prospective carrier parents are not aware of screening programs, though, upon taking the test, they appreciated knowing their risk thereby recognizing the test's importance [32]. There are many benefits in combining an extended carrier screening test with a NIPT. Firstly, a single comprehensive prenatal screening test is more efficient, reduces parental anticipation and anxiety, and provides results faster. Secondly, only a minority of couples undergo preconception carrier screening, even in countries with the highest level of healthcare provision. Thus, providing a comprehensive solution as the one presented here allows for screening a greater proportion of the potentially affected population. Thirdly, there is higher awareness for NIPT than carrier screening and the uptake of NIPT is constantly increasing, therefore, carrier screening as part of NIPT is more effective. Last but not least, the cost of a comprehensive NIPT compared to carrier screening is significantly lower. In order to maximize the test's clinical utility, economic implications should also be considered. Even though the cost of a screening test is more easily amortized, compared to the cost of treating an affected child [33, 34], a lower test cost allows for greater uptake. Our assay's targeted design significantly reduces the number of required reads, resulting in higher efficiency and scalability $[7,35]$. By combining all these features we can provide a cost-effective, extended carrier screening and NIPT solution to prospective parents, thereby extending the scope of prenatal care.

\section{Conclusions}

We present, for the first time, the development and validation of a highly accurate single and comprehensive NIPT for aneuploidies, microdeletions and a high number of monogenic diseases opening a new chapter in the field of Non-Invasive Prenatal Screening. The test presented here is based on a target capture enrichment and utilizes specific TACS designed to interrogate selected regions on chromosomes 13, 18, 21, X, Y, critical regions of 22q11.2, 1p36, Wolf-Hirschhorn, Smith-Magenis microdeletion syndromes and locations of known causative mutations involved in 50 autosomal recessive disorders, including life threatening conditions, moderate or severe impairments, congenital anomalies, developmental delay, hearing loss, blindness and metabolic disorders. By leveraging the inherent high enrichment uniformity and read depth of the 
assay in combination with multi-engine bioinformatics, we achieved higher than 99\% accuracy for the tested diseases, offering the same technological advantages, fast turnaround time, unrivalled classification and accurate fetal fraction estimation as previously described [7].

\section{Methods}

\section{Sample collection}

Protocols used for sample collection were approved by the Cyprus National Bioethics Committee (ЕEBK/EП/ 2011/14) and informed consent was obtained from all participants. The study was performed in accordance with the ethical standards of the institutional and/or national research committee and with the 1964 Helsinki Declaration.

For the retrospective validation of the comprehensive NIPT, a total of 2033 samples of known status (normal or abnormal for aneuploidy and microdeletion syndromes) were used which included cfDNA samples isolated from pregnant women with moderate to high risk pregnancies and genomic DNA isolated from the biological fathers. cfDNA was isolated from maternal peripheral blood samples $(8 \mathrm{ml})$ collected from pregnant women of at least 18 years of age, referred for NIPT from the 10th week of gestation onwards, in BCT StreckTubes (Streck Inc., Omaha, NE). A mean of $4 \mathrm{ml}$ plasma was isolated from all maternal peripheral blood samples via a double centrifugation protocol as previously described [7]. Genomic DNA from the biological father was isolated from self-collected buccal swab samples in 'hDNA free' FLOQSwabs (COPAN, Italy) following provided instructions.

For the retrospective prenatal diagnosis validation study, 200 previously characterized amniotic and CVS samples were sent to NIPD Genetics by collaborating laboratories.

\section{DNA isolation}

Circulating cfDNA was extracted from $4 \mathrm{ml}$ plasma using the QIAsymphony SP and the Qiasymphony Circulating DNA kit (Qiagen, Hilden, Germany) following manufacturer's instructions. Genomic DNA was isolated from buccal swabs, amniotic fluid or CVS samples using the QIAamp Mini kit (Qiagen) following manufacturer's instructions.

\section{Validation}

For the single comprehensive NIPT validation study, the status of all 2033 samples was known. All abnormal samples were previously confirmed by QF-PCR and/or complete chromosomal analysis by Chromosomal Microarray Analysis (CMA) or conventional karyotyping by the collaborating centers, after invasive procedures. At the time of testing the parental carrier status of all
2033 samples was unknown to those handling the samples (Table 1). Thus, the identification of normal constitution or detection of targeted mutations using the single comprehensive NIPT was confirmed on parental genomic DNA using Sanger sequencing. Prior to the study the samples identity was coded to allow for a blind validation study.

This comprehensive NIPT includes prenatal diagnosis confirmation for all high-risk NIPT samples (Additional file 1). Towards this goal, a retrospective validation study was performed using fetal tissues, i.e. amniotic fluid or chorionic villus sampling (CVS). In total, an independent set of 200 amniotic fluid or CVS samples were tested (Table 2). All 200 samples had been previously analyzed using standard prenatal diagnosis methodologies, including cytogenetic analysis and CMA. The sample identity was coded to allow for a blind validation study.

\section{Selection of 50 single-gene diseases for prenatal screening}

The 50 monogenic autosomal recessive diseases included in the NIPT panel were selected to provide clinical utility and prognostic value in prenatal screening (Additional file 2). They are all associated with a moderate to severe clinical phenotype. These include life-threatening conditions or conditions leading to moderate or severe impairments, congenital anomalies, developmental delay, hearing loss, blindness, metabolic disorders, etc. Although in several cases these conditions may be rare in the general (world-wide) population, they are encountered frequently in the high risk populations.

Only known pathogenic, or likely pathogenic variants were included during the selection of causative mutations (Additional file 4). In cases where a database dedicated specifically to the condition of interest was available, for example the cystic fibrosis database (The Clinical and Functional TRanslation of CFTR (CFTR2); available at http://cftr2.org; true on 27/11/2018), it was consulted in conjunction with references therein to obtain or deduce the detection frequencies for individual mutations in specific populations. Where no databases were available, a thorough review of the literature was performed for the selection of variants which have a clear correlation with the disease in specific populations (e.g. Tay Sachs, abetalipoproteinemia and Canavan disease in Ashkenazi Jews).

In total, 496 causative mutations are targeted, located on 49 genes with cumulative disease detection frequency higher than $70 \%$ (Additional file 4). For conditions where a very large number of mutations has been reported and most of the mutations tend to be rare and private/familial, such as 3-methylcrotonyl-CoA carboxylase deficiency, the most common pathogenic variants 
were selected, providing the highest detection frequency in the corresponding populations.

\section{Sequencing library preparation}

The cfDNA was subjected to library preparation as previously described [7] with modifications. In brief, initially blunt ending and 5' phosphorylation was performed using T4 polymerase and T4 kinase respectively. Sequencing adaptors were then ligated at both ends using T4 Ligase (New England Biolabs, Ipswich, UK). Nicks were removed in a fill-in reaction using Bst polymerase (New England Biolabs). Library amplification was performed using Herculase II Fusion Polymerase (Agilent Technologies, Santa Clara, $\mathrm{CA})$, and unique barcodes were assigned to all samples. At each step, products were purified using Ampure XP magnetic beads according to manufacturer's instructions.

Prior to library construction of buccal swab, amniotic fluid and CVS samples, extracted genomic DNA was sheared to an average size of $250 \mathrm{bp}$ using the Bioruptor Pico sonication system (Diagenode, Liege, Belgium). Blunt ending, adaptor ligation and adaptor fill-in reactions were performed without intermediate purification steps. A single purification step was performed following library amplification using Ampure XP magnetic beads according to manufacturer's instructions.

All library preparation steps were run on Hamilton STAR (Hamilton, Bonaduz, Switzerland) or epMotion (Eppendorf) systems using in-house developed automated methods.

\section{Design and preparation of target-capture sequences}

TArget Capture Sequences (TACS) were used to enrich regions of interest on chromosomes 13, 18, 21, X, Y and critical regions of 22q11.2, 1p36, Wolf-Hirschhorn and Smith-Magenis microdeletion syndromes. TACS were also specifically designed based on genomic locations of known causative mutations involved in 50 autosomal recessive disorders. Target loci were selected based on GC content, distance from repetitive elements and absence of known surrounding complex architecture [7].

For the preparation of TACS, PCR was performed using MyTaq polymerase (Bioline, London, UK) using normal DNA followed by purification using Ampure XP magnetic beads. TACS were quantified using the NanoDropND8000 (Thermo Scientific, Wilmington, DE, USA) and were pooled equimolarly. The final mix was blunt-ended using the Quick Blunting kit (New England Biolabs) and biotinylated using the Quick Ligation kit (New England Biolabs). Products were purified using Ampure XP magnetic beads.

\section{In-solution hybridization and sequencing}

Each amplified library was mixed with $2 \times$ hybridization buffer (Agilent Technologies), 10× blocking agent
(Agilent Technologies), blocking oligonucleotides, Cot1 DNA (Invitrogen, Carlsbad, CA), and salmon sperm DNA (Invitrogen). The hybridization reaction mix was denatured at $95^{\circ} \mathrm{C}$ for $3 \mathrm{~min}$ followed by blocking incubation at $37^{\circ} \mathrm{C}$ before being added to the biotinylated TACS. The samples were then incubated at $65^{\circ} \mathrm{C}$ for $16 \mathrm{~h}$. After hybridization, unbound DNA was washed and captured sequences were eluted by heating as previously described [7]. All capture steps were performed on a Hamilton STARlet liquid handler using in-house developed methods.

Enriched samples were then amplified using outerbound primers, pooled equimolarly and sequenced using a NextSeq 500 sequencer (illumina).

\section{Data analysis}

The sequencing data of the enriched samples was analyzed using methods as described in Koumbaris et al. with modifications [7]. The cfDNA and paternal genomic DNA samples were further processed to compute the parental carrier status and subsequently determine the fetal risk for the targeted monogenic diseases. For parental carrier status determination, the Variant Allele Frequency (VAF) at each targeted locus was used. Specifically, we used the number of times the predefined variant allele was sequenced over the total number of times the locus was sequenced (Additional file 5).

For the paternal sample (buccal swab), the expected VAF for heterozygous loci (carrier) and homozygous loci is 0.5 and 1 respectively. In maternal samples, the expected value of the VAF is dependent on the cell free maternal fraction present in the plasma. Hence, a carrier mother is expected to have (i) a VAF value equal to half the maternal fraction if the fetus has only the wild type allele and (ii) a VAF value greater than half the maternal fraction if the fetus is either heterozygous or homozygous for the variant allele. The algorithm estimates the carrier status of each of the parents using proportions tests (Additional file 5).

The fetal risk for inheriting the targeted genetic condition was determined from the estimated carrier status of the parents following the Mendelian law of inheritance. Specifically, if both parents are carriers for the same recessive genetic condition, then the fetus has a $25 \%$ chance of inheriting the genetic condition. In such cases the pregnancy would be considered as "high risk" for the monogenic disease. All amniotic fluid and CVS samples were processed using the bioinformatics pipeline previously described [7]. Copy number estimation for all targeted regions is achieved using data normalization and change-point detection methods [7, 36] . 


\section{Supplementary information}

Supplementary information accompanies this paper at https://doi.org/10. 1186/s13039-019-0459-8.

Additional file 1: Figure S1. Workflow of the new single comprehensive NIPT for aneuploidies, microdeletions and single gene diseases. (Left side) Cell-free fetal DNA (cfDNA) is analyzed for fetal risk determination for chromosomal aneuploidies (trisomy 21, trisomy 18 and trisomy 13), sex chromosome aneuploidies (SCA) and four microdeletion syndromes using a custom multi-engine bioinformatics analysis pipeline. In the same assay, cell-free maternal (cfmDNA) and genomic DNA from the biological father are subjected in-solution hybridization enrichment for parental carrier status determination for 50 autosomal recessive conditions. The fetal risk for inheriting a genetic condition is determined following the Mendelian law of inheritance. A pregnancy is considered as "high risk" if both parents are carriers of the same autosomal recessive disease. (Right side) Following a high risk NIPT result for an aneuploidy or microdeletion or single gene diseases prenatal diagnosis is offered by analysis of amniotic fluid or CVS.

Additional file 2: Table S1. List of 50 monogenic disorders included in the targeted disease panel.

Additional file 3: Table S2. The theoretical and estimated risk for the parents and the fetus.

Additional file 4: Table S3. List of 50 monogenic diseases screened by comprehensive NIPT. Targeted mutations covered are outlined along with detection frequencies in corresponding populations

Additional file 5: Figure S2. Flowchart illustrating the bioinformatics analysis pipeline for a typical sequencing run consisting of plasma samples. The same pipeline applies for paternal samples with the last step being performed for variant calling (monogenic diseases) only.

\section{Abbreviations}

cfDNA: Cell-free DNA; cffDNA: Cell-free fetal DNA; cfmDNA: Cell-free maternal DNA; CPM: Confined Placental Mosaicism; FN: False Negative; FP: False Positive; NGS: Next Generation Sequencing; NIPT: Non-invasive prenatal testing; SCA: Sex chromosome aneuploidies; TACS: Target Capture sequences

\section{Acknowledgements}

Not applicable.

\section{Authors' contributions}

GK designed and supervised the study and edited the manuscript. AA, PM and $\mathrm{CL}$ developed statistical models and bioinformatics analysis pipelines and analysed the data. MN performed the experiments and helped to edit the manuscript. Ml assisted in the design of the study, supervised the study and drafted the manuscript. EK oversaw the experimental workflow and performed results interpretation. KT assisted in the performance of experiments and provided helpful suggestions and troubleshooting. W, CS GC, PC, ME, IP and DS contributed in sample collection data and provided helpful suggestions and troubleshooting. PCP conceived, designed, coordinated and supervised the study. All authors read and approved the final manuscript.

\section{Funding}

Not applicable.

\section{Availability of data and materials}

All supporting data are included in the manuscript.

\section{Ethics approval and consent to participate}

The study and all protocols were approved by the I Cyprus National Bioethics Committee (EEBK/Eח/2011/14) and all participants provided a written informed consent prior to participation.

\section{Consent for publication}

Not applicable.

\section{Competing interests}

The authors, GK, AA, MN, CL, KT, EK, W, MI and PCP are employed by NIPD Genetics Ltd. The authors CS, GC, PC, ME, IP, DS declare that they have no competing interests. PM was employed by NIPD Genetics Public Company Ltd. at the time of the study but currently has no competing interests.

\section{Author details}

${ }^{1}$ NIPD Genetics Public Company Ltd, Neas Engomis 31, Nicosia 2409, Cyprus.

${ }^{2}$ The Cyprus Institute of Neurology and Genetics, International Airport Avenue, 6, Ayios Dometios, Nicosia 2370, Cyprus. ${ }^{3}$ Cyprus School of Molecular Medicine, International Airport Avenue, 6, Ayios Dometios, Nicosia 2370, Cyprus. ${ }^{4}$ Cytogenetics and Molecular Genetics Department, Bioiatriki Healthcare Group, Athens, Greece. ${ }^{5}$ Genotypos Science Labs, Athens, Greece.

${ }^{6}$ Access To Genome, Genetics Laboratory, Athens-Thessaloniki, Greece.

${ }^{7}$ Cytogenomic Medical Laboratory, Bucuresti, Romania.

Received: 10 June 2019 Accepted: 29 October 2019

Published online: 21 November 2019

\section{References}

1. Lo YM, Corbetta N, Chamberlain PF, Rai V, Sargent IL, Redman CW, et al. Presence of fetal DNA in maternal plasma and serum. Lancet. 1997; 350(9076):485-7.

2. Sparks AB, Wang ET, Struble CA, Barrett W, Stokowski R, McBride C, et al. Selective analysis of cell-free DNA in maternal blood for evaluation of fetal trisomy. Prenat Diagn. 2012;32(1):3-9.

3. Sparks AB, Struble CA, Wang ET, Song K, Oliphant A. Noninvasive prenatal detection and selective analysis of cell-free DNA obtained from maternal blood: evaluation for trisomy 21 and trisomy 18. Am J Obstet Gynecol. 2012; 206(4):319 e1-9.

4. Sehnert AJ, Rhees B, Comstock D, de Feo E, Heilek G, Burke J, et al. Optimal detection of fetal chromosomal abnormalities by massively parallel DNA sequencing of cell-free fetal DNA from maternal blood. Clin Chem. 2011; 57(7):1042-9.

5. Nicolaides KH, Syngelaki A, Gil M, Atanasova V, Markova D. Validation of targeted sequencing of single-nucleotide polymorphisms for non-invasive prenatal detection of aneuploidy of chromosomes 13, 18, 21, X, and $Y$. Prenat Diagn. 2013:33(6):575-9.

6. Liao GJ, Lun FM, Zheng YW, Chan KC, Leung TY, Lau TK, et al. Targeted massively parallel sequencing of maternal plasma DNA permits efficient and unbiased detection of fetal alleles. Clin Chem. 2011;57(1):92-101.

7. Koumbaris G, Kypri E, Tsangaras K, Achilleos A, Mina P, Neofytou M, et al. Cell-free DNA analysis of targeted genomic regions in maternal plasma for non-invasive prenatal testing of trisomy 21 , trisomy 18 , trisomy 13 , and fetal sex. Clin Chem. 2016:62(6):848-55.

8. Fan HC, Blumenfeld YJ, Chitkara U, Hudgins L, Quake SR. Noninvasive diagnosis of fetal aneuploidy by shotgun sequencing DNA from maternal blood. Proc Natl Acad Sci U S A. 2008;105(42):16266-71.

9. Chiu RW, Chan KC, Gao Y, Lau VY, Zheng W, Leung TY, et al. Noninvasive prenatal diagnosis of fetal chromosomal aneuploidy by massively parallel genomic sequencing of DNA in maternal plasma. Proc Natl Acad Sci U S A. 2008;105(51):20458-63.

10. Gregg AR, Skotko BG, Benkendorf JL, Monaghan KG, Bajaj K, Best RG, et al. Noninvasive prenatal screening for fetal aneuploidy, 2016 update: a position statement of the American College of Medical Genetics and Genomics. Genet Med. 2016:18(10):1056-65.

11. Benn P, Borrell A, Chiu RW, Cuckle H, Dugoff L, Faas B, et al. Position statement from the chromosome abnormality screening committee on behalf of the Board of the International Society for prenatal diagnosis. Prenat Diagn. 2015:35(8):725-34

12. Committee Opinion No. 640: Cell-free DNA Screening for Fetal Aneuploidy. Obstet Gynecol. 2015:126:e31-7.

13. Gonzalez-Gonzalez MC, Garcia-Hoyos M, Trujillo MJ, Rodriguez de Alba M, Lorda-Sanchez I, Diaz-Recasens J, et al. Prenatal detection of a cystic fibrosis mutation in fetal DNA from maternal plasma. Prenat Diagn. 2002;22(10):946-8

14. Chiu RW, Lau TK, Leung TN, Chow KC, Chui DH, Lo YM. Prenatal exclusion of beta thalassaemia major by examination of maternal plasma. Lancet. 2002:360(9338):998-1000

15. Amicucci P, Gennarelli M, Novelli G, Dallapiccola B. Prenatal diagnosis of myotonic dystrophy using fetal DNA obtained from maternal plasma. Clin Chem. 2000;46(2):301-2

16. Perlado S, Bustamante-Aragones A, Donas M, Lorda-Sanchez I, Plaza J, Rodriguez de Alba M. Fetal genotyping in maternal blood by digital PCR: 
towards NIPD of monogenic disorders independently of parental origin. PLoS One. 2016;11(4):e0153258.

17. Lun FM, Tsui NB, Chan KC, Leung TY, Lau TK, Charoenkwan P, et al. Noninvasive prenatal diagnosis of monogenic diseases by digital size selection and relative mutation dosage on DNA in maternal plasma. Proc Natl Acad Sci U S A. 2008;105(50):19920-5.

18. Lam KW, Jiang P, Liao GJ, Chan KC, Leung TY, Chiu RW, et al. Noninvasive prenatal diagnosis of monogenic diseases by targeted massively parallel sequencing of maternal plasma: application to beta-thalassemia. Clin Chem. 2012;58(10):1467-75.

19. Chitty LS, Mason S, Barrett AN, McKay F, Lench N, Daley R, et al. Noninvasive prenatal diagnosis of achondroplasia and thanatophoric dysplasia: next-generation sequencing allows for a safer, more accurate, and comprehensive approach. Prenat Diagn. 2015;35(7):656-62.

20. Camunas-Soler J, Lee H, Hudgins L, Hintz SR, Blumenfeld YJ, El-Sayed YY, et al. Noninvasive prenatal diagnosis of single-gene disorders by use of droplet digital PCR. Clin Chem. 2018;64(2):336-45.

21. Barrett AN, McDonnell TC, Chan KC, Chitty LS. Digital PCR analysis of maternal plasma for noninvasive detection of sickle cell anemia. Clin Chem. 2012;58(6):1026-32.

22. New Ml, Tong YK, Yuen T, Jiang P, Pina C, Chan KC, et al. Noninvasive prenatal diagnosis of congenital adrenal hyperplasia using cell-free fetal DNA in maternal plasma. J Clin Endocrinol Metab. 2014;99(6):E1022-30.

23. Ma D, Ge H, Li X, Jiang T, Chen F, Zhang Y, et al. Haplotype-based approach for noninvasive prenatal diagnosis of congenital adrenal hyperplasia by maternal plasma DNA sequencing. Gene. 2014;544(2):252-8.

24. Jenkins LA, Deans ZC, Lewis C, Allen S. Delivering an accredited noninvasive prenatal diagnosis service for monogenic disorders and recommendations for best practice. Prenat Diagn. 2018;38(1):44-51.

25. Luo Y, Jia B, Yan K, Liu S, Song X, Chen M, et al. Pilot study of a novel multifunctional noninvasive prenatal test on fetus aneuploidy, copy number variation, and single-gene disorder screening. Mol Genet Genom Med. 2019; 7(4):e00597.

26. American College of O, Gynecologists Committee on G. Committee Opinion No. 545: Noninvasive prenatal testing for fetal aneuploidy. Obstet Gynecol. 2012;120(6):1532-4.

27. Zhang J, Li J, Saucier JB, Feng Y, Jiang Y, Sinson J, et al. Non-invasive prenatal sequencing for multiple Mendelian monogenic disorders using circulating cell-free fetal DNA. Nat Med. 2019;25:701-2.

28. Li B, Sahota DS, Lao Tा, Xu J, Hu SQ, Zhang L, et al. Applicability of firsttrimester combined screening for fetal trisomy 21 in a resource-limited setting in mainland China. BJOG. 2016;123(Suppl 3):23-9.

29. Gil MM, Revello R, Poon LC, Akolekar R, Nicolaides KH. Clinical implementation of routine screening for fetal trisomies in the UK NHS: cellfree DNA test contingent on results from first-trimester combined test. Ultrasound Obstet Gynecol. 2016;47(1):45-52.

30. Edwards JG, Feldman G, Goldberg J, Gregg AR, Norton ME, Rose NC, et al. Expanded carrier screening in reproductive medicine-points to consider: a joint statement of the American College of Medical Genetics and Genomics, American College of Obstetricians and Gynecologists, National Society of genetic counselors, perinatal Quality Foundation, and Society for MaternalFetal Medicine. Obstet Gynecol. 2015;125(3):653-62.

31. Tanner AK, Valencia CA, Rhodenizer D, Espirages M, Da Silva C, Borsuk L, et al. Development and performance of a comprehensive targeted sequencing assay for pan-ethnic screening of carrier status. J Mol Diagnost. 2014;16(3):350-60

32. Tardif J, Pratte A, Laberge AM. Experience of carrier couples identified through a population-based carrier screening pilot program for four founder autosomal recessive diseases in Saguenay-lac-saint-Jean. Prenat Diagn. 2018;38(1):67-74.

33. Lazarin GA, Haque IS, Nazareth S, lori K, Patterson AS, Jacobson JL, et al. An empirical estimate of carrier frequencies for $400+$ causal Mendelian variants: results from an ethnically diverse clinical sample of 23,453 individuals. Genet Med. 2013;15(3):178-86.

34. Chiu EKL, Hui WWI, Chiu RWK. cfDNA screening and diagnosis of monogenic disorders - where are we heading? Prenat Diagn. 2018; 38(1):52-8.

35. Neofytou MC, Tsangaras K, Kypri E, Loizides C, loannides M, Achilleos A, et al. Targeted capture enrichment assay for non-invasive prenatal testing of large and small size sub-chromosomal deletions and duplications. PLOS One. 2017;12(2):e0171319.
36. Cleynen A, Koskas M, Lebarbier E, Rigaill G, Robin S. Segmentor3lsBack: an R package for the fast and exact segmentation of Seq-data. Algorithms Mol Biol. 2014;9(1):6

\section{Publisher's Note}

Springer Nature remains neutral with regard to jurisdictional claims in published maps and institutional affiliations.
Ready to submit your research? Choose BMC and benefit from:

- fast, convenient online submission

- thorough peer review by experienced researchers in your field

- rapid publication on acceptance

- support for research data, including large and complex data types

- gold Open Access which fosters wider collaboration and increased citations

- maximum visibility for your research: over $100 \mathrm{M}$ website views per year

At BMC, research is always in progress.

Learn more biomedcentral.com/submissions 